\title{
Case Report: Extra-Pharyngeal Ingested Foreign Body (Fish Bone)
}

\author{
Daniel Kim ${ }^{1}$ and Geoffrey Young ${ }^{2 *}$ \\ ${ }^{1}$ Mayo Clinic Florida, Jacksonville, FL, USA \\ ${ }^{2}$ Miami Cancer Institute, Miami, FL, USA

\section{Introduction}

Foreign body ingestion is a common clinical problem for otorhinolaryngology. Fish bone ingestion poses additional hazards such as increased chance of impaction. If the fish bone passes into the gastrointestinal tract, there has been documentation of potential bowel perforation [1]. The diagnosis of a foreign body within the oroesophageal tract will frequently include a history of ingestion, with subsequent chest or throat discomfort, gagging, emesis, or dysphagia [2]. Typically, if the fish bone does become impacted, endoscopic measures are able to retrieve the object [3]. We present a patient with a history of fish bone ingestion and impaction, requiring operative management for retrieval.

\section{Case Report}

A 65-year-old male was admitted to the emergency department after complaints of continuous throat pain and concern for airway occlusion. The patient reported eating fish 5 days prior to his admission. He was initially evaluated at an outside hospital. The patient was afebrile, but was found to have a leukocytosis. A CT of the neck was performed, which revealed signs of a fishbone extending from the proximal esophagus into the left lateral soft tissue with evidence of surrounding abscess. An esophagogastroduodenoscopy and flexible laryngoscopy were performed. Both were unable to remove the ingested fishbone. The patient was subsequently transferred to our facility for further evaluation. A repeat CT neck revealed a fishbone extending from the proximal cervical esophagus at approximately C4-C5 level with a $2.2 \mathrm{~cm}$ abscess involving the left retropharyngeal space (Figure 1).

The patient was taken to the operating room, where a direct laryngoscopy and rigid esophagoscopy was first attempted. The fish bone was unable to be visualized.
We concluded that the fish bone had migrated through the posterior pharynx into the neck, and elected to proceed with a left neck exploration. An incision was made along an existing skin crease. After a subplatysmal flap was raised, the anterior border of the sternocleidomastoid was dissected down to the level of the internal jugular vein. The preoperative CT scan indicated the fish bone was at the level of the carotid bifurcation. Fibrinous inflammatory material was discovered, but no foreign body was visualized. Intraoperative fluoroscopy was used in an attempt to locate the cervical level of the fish bone. Both superior and lateral aspects failed to identify a radiopaque foreign body. We then continued dissection by mobilizing the hypopharynx off the prevertebral fascia. The fish bone was identified postcricoid, having migrated through the posterior pharyngeal wall. The specimen was sent to pathology and cultures were taken of the fibrinous inflammatory material (Figure 2). The area of dissection was irrigated and hemostasis was achieved. A JP drain was placed and put to bulb suction. Extubation of this patient was uneventful. The patient was placed on a clear liquid diet for 5 days and advanced to a soft diet afterwards. The patient was discharged on post-operative day one without complications. The patient resumed a regular diet within two weeks.

\section{Discussion}

Ingestion of foreign bodies is common. Fish bones are a common foreign body that can be impacted within the oroesophageal tract given their slender

*Corresponding author: Geoffrey Young, Miami Cancer Institute, Miami, FL, USA

Accepted: October 21, 2019

Published online: October 23, 2019

Citation: Kim D, Young G (2019) Case Report: Extra-Pharyngeal Ingested Foreign Body (Fish Bone). J Head Neck Surg 2(1):42-44 


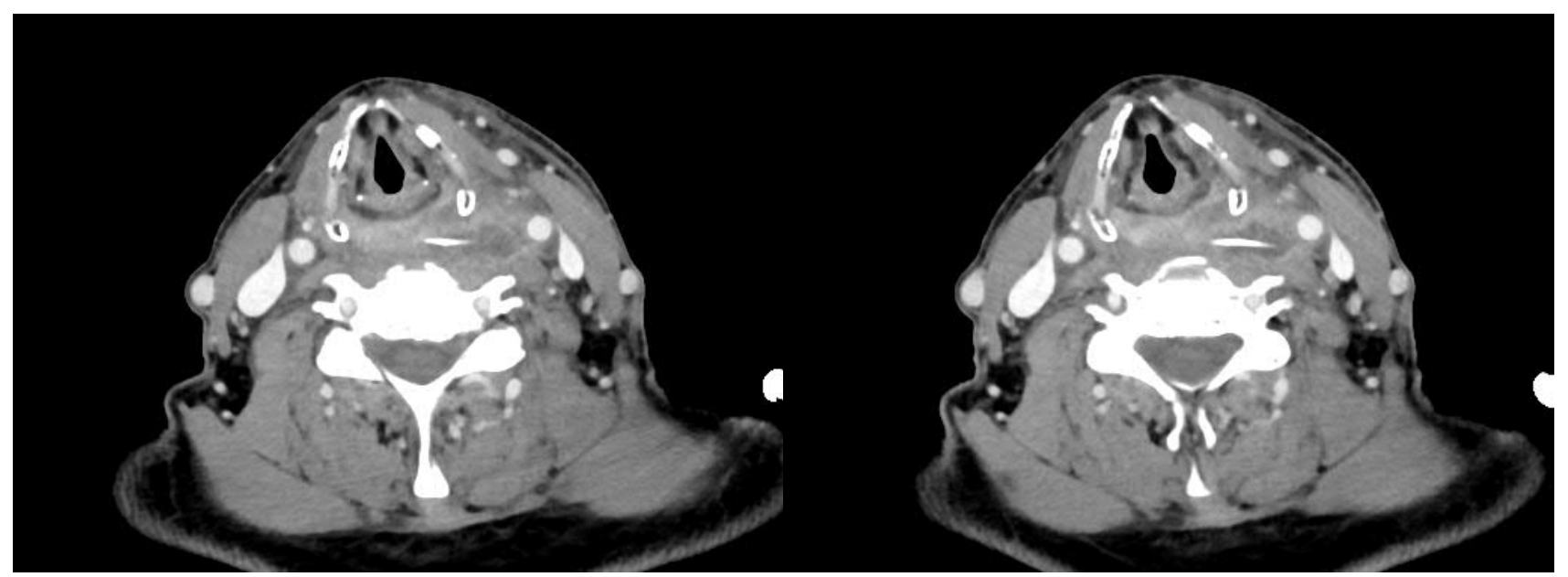

Figure 1: The fish bone is seen penetrating the esophagus into the retropharyngeal space with associated abscess formation.

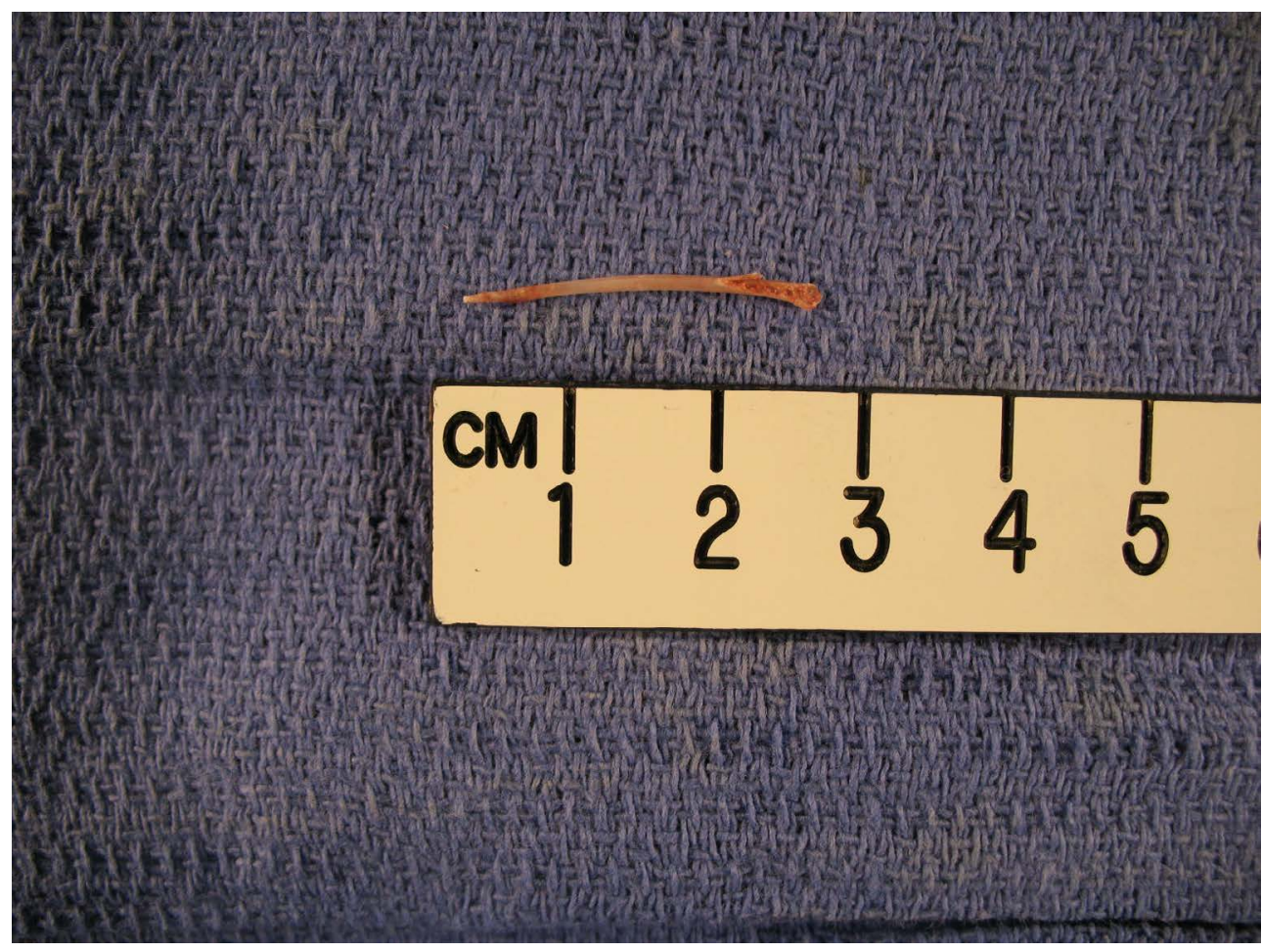

Figure 2: Fish bone measuring $2.5 \mathrm{~cm}$ in length $\times 0.1 \mathrm{~cm}$ in diameter.

shape and sharp points. Most are impacted within the tonsillar pillars or base of tongue [4]. By and large, these are able to be retrieved either by laryngoscopy or endoscopy. Ngan, et al. reported a prospective study regarding their institution's experience with fish bone ingestion. They report an $88 \%$ rate of successful removal under either direct or endoscopic means [3]. If the fish bone passes into the gastrointestinal tract, perforation is uncommon and less than $1 \%$ of these will perforate the bowel [1]. The ileum is the most common site of perforation [1].

The usual protocol in the diagnosis of a foreign body includes a lateral view plain radiograph. However, this has been shown to be unreliable. Plain radiography may not reveal a fish bone due to their variable radiopacity. Lue, et al. demonstrated a sensitivity and specificity of $39 \%$ and $72 \%$ when using plain radiography to evaluate fish bone foreign bodies in cadaver head and neck specimens [5].

A CT scan is useful in the evaluation of a patient with a history of an impacted fish bone. Eliashar reported a prospective study concluding that a CT is a reliable method for diagnosing esophageal bone impaction [6]. Akazawa, et al. reviewed 76 patients with suspected 
esophageal impaction of fish bones. They concluded a sensitivity and specificity of $100 \%$ for the presence of an impacted fish bone [7]. Of note, they reported a sensitivity and specificity of $54.8 \%$ and $100 \%$ respectively when using plain $x$-ray in the evaluation of a suspected fish bone impaction [7]. The use of computed tomography as a pre-operative evaluation in our case was useful in confirming the continued impaction of the fish bone. However, using the cervical vertebrae as a landmark from the preoperative CT scan may not be useful, as the fishbone may move in relation to the vertebrae upon positioning of the head and neck during an operation.

There have been cases in the literature documenting migration of a fish bone through the esophagus. A retained fish bone may cause deep neck abscesses, as in our case report. Furthermore, case reports exist of severe complications caused by fish bones migrating towards the aorta, or penetrating the pericardium causing cardiac tamponade $[8,9]$. Migration should be suspected in cases where there is a positive neck radiograph and a negative esophagoscopy [10].

Based on our experience with fish bone ingestion, a computed tomography is recommended to confirm the diagnosis of an impacted fish bone. Exploration for a migrated fish bone requires meticulous dissection and technique. Magnifying loupes are useful adjuncts during exploration.

\section{References}

1. Hsu SD, DC Chan, YC Liu (2005) Small-bowel perforation caused by fish bone. World J Gastroenterol 11: 1884-1885.

2. Brooks JW (1972) Foreign bodies in the air and food passages. Ann Surg 175: 720-732.

3. Ngan JH, Fok PJ, Lai EC, et al. (1990) A prospective study on fish bone ingestion. Experience of 358 patients. Ann Surg 211: 459-462.

4. Leong HK, RK Chan (1987) Foreign bodies in the upper digestive tract. Singapore Medical Journal 28: 162-165.

5. Lue AJ, WD Fang, S Manolidis (2000) Use of plain radiography and computed tomography to identify fish bone foreign bodies. Otolaryngol Head Neck Surg 123: 435-438.

6. Eliashar R, Dano I, Dangoor E, et al. (1999) Computed tomography diagnosis of esophageal bone impaction: A prospective study. Ann Otol Rhinol Laryngol 108: 708-710.

7. Akazawa $Y$, Watanabe $S$, Nobukiyo $S$, et al. (2004) The management of possible fishbone ingestion. Auris Nasus Larynx 31: 413-416.

8. Alam AM, Shuaib IL, Hock LC, et al. (2005) Perforation of oesophagus and aorta by an unusual migratory fish bone: Case report. Nepal Med Coll J 7: 150-151.

9. Sharland MG, BC McCaughan (1993) Perforation of the esophagus by a fish bone leading to cardiac tamponade. Ann Thorac Surg 56: 969-971.

10. Chee LW, DS Sethi (1999) Diagnostic and therapeutic approach to migrating foreign bodies. Ann Otol Rhinol Laryngol 108: 177-180.

DOI: $10.36959 / 605 / 536$

Copyright: (C) $2019 \mathrm{Kim} \mathrm{D,} \mathrm{et} \mathrm{al.} \mathrm{This} \mathrm{is} \mathrm{an} \mathrm{open-access} \mathrm{article} \mathrm{distributed} \mathrm{under} \mathrm{the} \mathrm{terms} \mathrm{of}$ the Creative Commons Attribution License, which permits unrestricted use, distribution, and reproduction in any medium, provided the original author and source are credited. 